\title{
RSRM TP-H1148 Main Grain Propellant Crack Initiation Evaluation
}

\author{
Todd E. Earnest ${ }^{*}$ \\ ATK Thiokol, Brigham City, UT, 84302
}

\begin{abstract}
Pressurized TP-H1148 propellant fracture toughness testing was performed to assess the potential for initiation of visually undetectable cracks in the RSRM forward segment transition region during motor ignition. Two separate test specimens were used in this evaluation. Testing was performed in cold-gas and hot-fire environments, and under both static and dynamic pressurization conditions. Analysis of test results demonstrates safety factors against initiation of visually undetectable cracks in excess of 8.0.
\end{abstract}

$\begin{array}{ll}\text { RSRM } & \text { Reusable Solid Rocket Motor } \\ \text { PBAN } & =\text { Polybutadiene-acrylic acid-acrylonitrile } \\ M D F S & =\text { Minimum Detectable Flaw Size } \\ C D A & =\text { Critical Defect Assessment } \\ G_{C} & =\text { Critical Strain Energy Release Rate, or Fracture Toughness } \\ G_{i} & =\text { Induced Strain Energy Release Rate } \\ \eta & =\text { Geometry Factor } \\ B & =\text { Specimen Thickness } \\ P & =\text { Load as a Function of Displacement } \\ \delta & =\text { Specimen Displacement } \\ \delta_{c} & =\text { Specimen Displacement at Crack Initiation } \\ S F & =\text { Safety Factor } \\ F E & =\text { Finite Element }\end{array}$

\section{Introduction}

$\mathrm{T}$ HE Reusable Solid Rocket Motor (RSRM) forward segment is cast with PBAN propellant (TP-H1148) to form an 11-point star configuration that transitions to a tapered center perforated bore (see Figure 1). The geometry of the transition region between the fin valleys and the bore causes a localized area of high strain during horizontal storage. Updated analyses using worst-case mechanical properties at $40^{\circ} \mathrm{F}$ and improved modeling techniques indicated a slight reduction in safety margins over previous predictions. Although there is no history of strain induced cracks or flaws in the transition region propellant, a proactive test effort was initiated to better understand the implications of the new analysis, primarily the resistance of TP-H1148 propellant to crack initiation ${ }^{\dagger}$ during RSRM ignition.

\section{A. Minimum Detectable Flaw Size}

Previous ATK Thiokol testing was performed to statistically define the threshold for visual detection of cracks or flaws on cast surfaces of TP-H1148 propellant. From that testing, a minimum detectable flaw size (MDFS) specific to the RSRM forward segment transition region propellant was determined. The MDFS is defined as the minimum flaw size that will be detected during visual inspection with 95 percent confidence and 90 percent probability. As such, the MDFS provides a prediction of the largest potential crack length that can be missed during inspection. The MDFS determined for the transition region propellant was less than 0.10 " length, and was the smallest crack size considered in assessing the resistance of TP-H1148 propellant to crack initiation.

\footnotetext{
* Design Engineer, Ballistics and Motor Performance, 9160 N. Highway 83/LD1, AIAA Member.

+ Crack initiation refers to the time during which extension of a preexisting crack just begins to occur. Crack initiation within the context of this paper does not refer to formation of a new, previously nonexistent crack.

Not yet approved for public release

(C2005 ATK Thiokol, Inc., an affiliate of Alliant Techsystems Inc.
}

American Institute of Aeronautics and Astronautics 


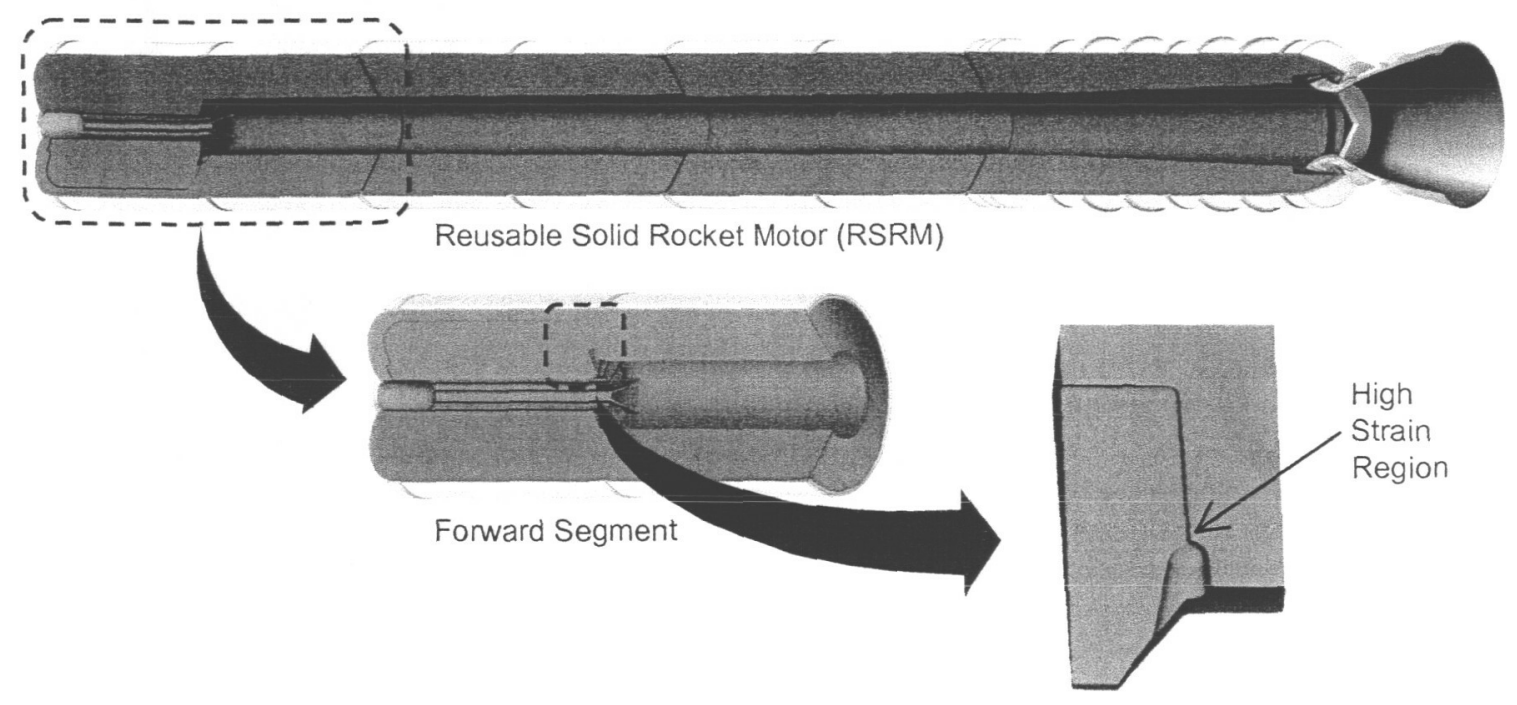

Transition Region Propellant

Figure 1 RSRM forward segment transition region propellant.

\section{B. Approach}

A multi-phase test approach was designed to assess the resistance of the transition region MDFS to crack initiation during RSRM ignition. Of particular interest was testing that evaluated the MDFS in the presence of combustion. Cold-gas and hot-fire testing of intentionally flawed TP-H1148 propellant specimens, under both static and dynamic pressurization conditions, was performed. Testing was designed to determine the fracture toughness $\left(G_{c}\right)$ of TP-H1148 propellant under the most structurally severe conditions, which occur during RSRM ignition. Fracture toughness results were compared to the analytically determined induced strain energy release rate $\left(G_{i}\right)$ of the MDFS to assess the margin against crack initiation during RSRM ignition. Further testing was performed to demonstrate the response of the transition region MDFS to a simulated RSRM ignition combustion environment.

\section{Test Specimen Configuration and Setup}

Two separate test specimens were used to evaluate the resistance of transition region MDFS to crack initiation during RSRM ignition: the center one-sided crack specimen, and the crack propagation chamber specimen.

\section{A. Center One-Sided Crack Specimen}

The center one-sided crack specimen, shown in Figure 2, was used to determine the fracture toughness of TPH1148 propellant at various pressures. The configuration of this specimen was optimized to provide plane strain fracture toughness for TP-H1148 propellant, and conforms to ASTM standards. The specimen consisted of a rectangular shaped piece of TPH1148 propellant of a constant thickness, with aluminum end tabs secondarily bonded on each end. A center groove with the initial crack at one end was machined through the entire specimen thickness. The initial crack, which also extended the entire specimen thickness, was precisely created using a razor blade configured in a CNC mill.

Testing was performed by mechanically loading the specimen in tension inside a statically pressurized Instron $^{(\mathbb{R}}$ chamber at predetermined crosshead rates through total specimen failure. The load required to cause the initial crack to just extend, or initiate, was used to analytically determine the fracture toughness. High-speed

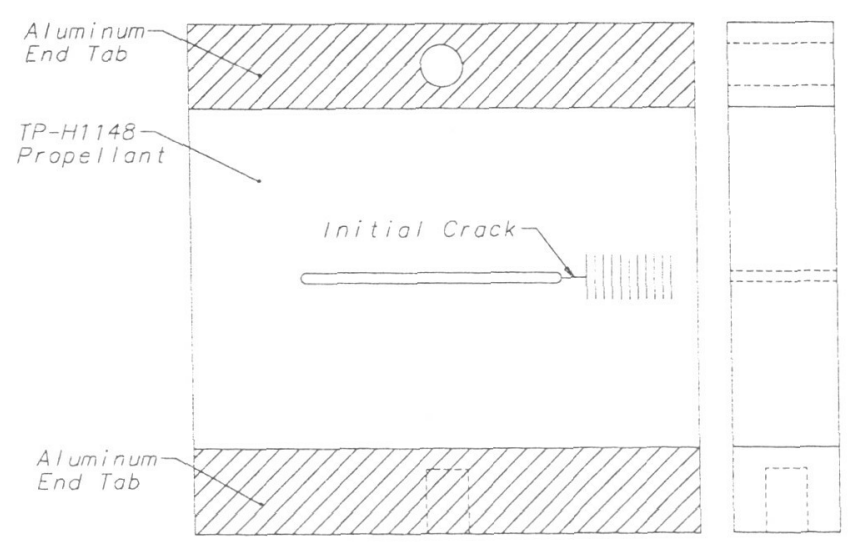

Figure 2 Center one-sided crack specimen. 
digital video was used to record the test events and to correlate critical test events with the load curve. Frame rates up to 2000 frames per second were used. The fracture toughness of TP-H1148 propellant using the center one-sided crack specimen was determined at multiple pressures and rates, including pressures and rates typical of RSRM ignition.

\section{B. Crack Propagation Chamber Specimen}

The crack propagation chamber specimen, shown in Figure 3, was also used to determine pressurized fracture toughness of TP-H1148 propellant. This specimen is a modified version of the crack propagation chamber specimen designed and used by the Critical Defect Assessment (CDA) program for evaluation of crack growth in another ATK PBAN propellant ${ }^{1}$. The CDA test program was based on testing performed at Penn State University for the Structural Ballistic Risk Assessment Methodology (SBRAM) program².

Loading of the crack propagation chamber specimen was accomplished through dynamic pressurization to simulate the RSRM ignition transient. Testing was performed in the crack propagation chamber, a nozzled window bomb test fixture designed to control propellant deformation, and thereby generate energy at the initial crack tip. The size of the initial crack in each propellant specimen was precisely controlled through use of a razor blade configured in a CNC mill. As is shown in Figure 3, the initial crack extended through the entire specimen thickness.

Cold-gas testing in the crack propagation chamber was performed by dynamically pressurizing the propellant specimen according to the RSRM ignition transient. This was accomplished through use of a nitrogen tank farm in combination with a pressurization rate-of-rise system. The rate-of-rise system allowed for exact control of specimen pressurization. High-speed digital video was used to record the events of each test and to correlate critical test events to the pressure trace during data analysis. Frame rates up to 2000 frames per second were used.

Hot-fire testing in the crack propagation chamber was accomplished by igniting the propellant specimen
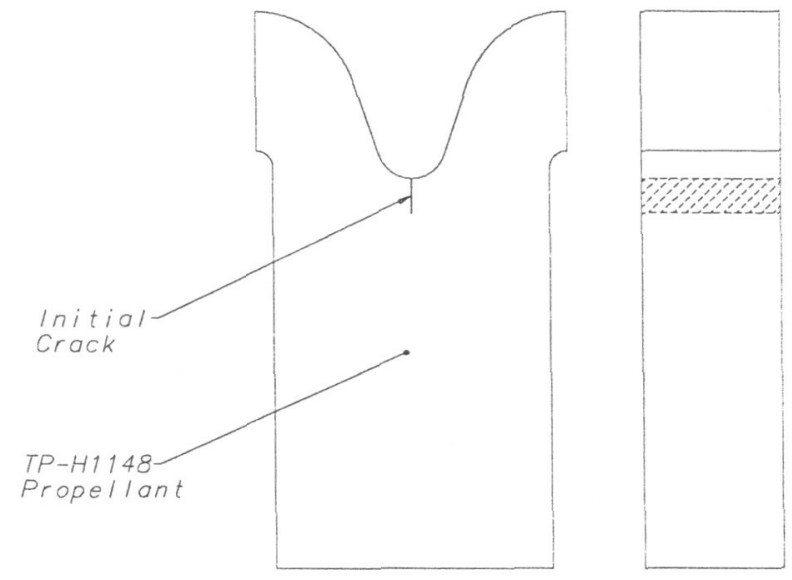

Figure 3 Crack propagation chamber specimen.

in a manner that simulated the RSRM ignition pressurization transient. Hot-fire pressurization was controlled, primarily, through sizing of the igniter charge and the nozzle exit diameter. High-speed digital video was again used to record the events of each test and to correlate critical test events to the pressure trace during data analysis. Frame rates up to 2000 frames per second were used.

Testing in the crack propagation chamber window bomb was performed using three separate specimen holders. The propellant specimen interfaced with the specimen holders as shown in Figure 4. As can be seen, a free volume exists between the sides of the propellant specimen and the walls of the specimen holder. This free volume controlled the amount of propellant deformation that occurred during specimen pressurization, which in turn controlled the amount of $\mathrm{G}_{\mathrm{i}}$ that was generated at the initial crack tip. The amount of $\mathrm{G}_{\mathrm{i}}$ generated at the crack tip increased as the specimen holder free volume increased. Various combinations of initial crack size and propellant specimen holder free volumes were tested, providing data to determine fracture toughness at several pressures and rates.

Acrylic window frames were placed on the front and back faces of the propellant specimen after the specimen was configured in the specimen holder. A grease interface between the propellant and the acrylic windows helped seal the test fixture during testing and inhibited undesired burning of the specimen during hotfire tests. Figure 5 is a photo of the test fixture setup showing the propellant specimen configured in the specimen holder with the acrylic windows in place.

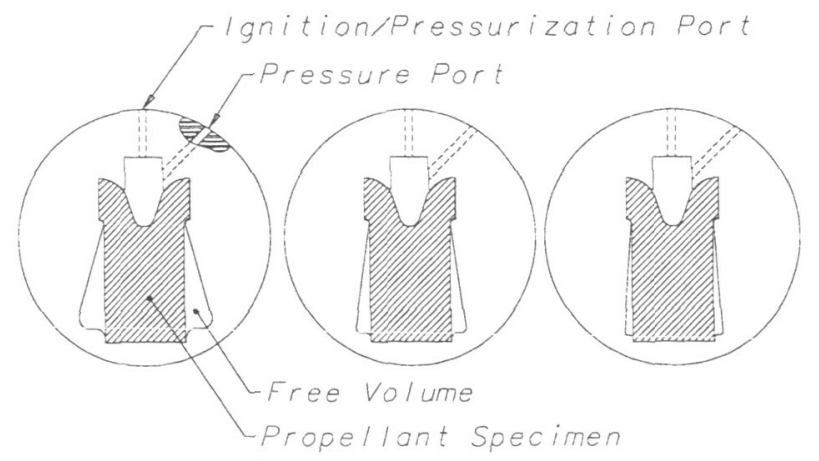

Figure 4 Crack propagation chamber specimen holders. 
Testing in a fourth specimen holder specifically designed to simulate $G_{i}$ of the transition region MDFS during RSRM ignition was also performed. Simulation of the MDFS $\mathrm{G}_{i}$ was controlled through analytical sizing of the specimen holder free volume. Specimen testing in this fourth holder provided a demonstration of the response of the MDFS to the RSRM ignition environment.

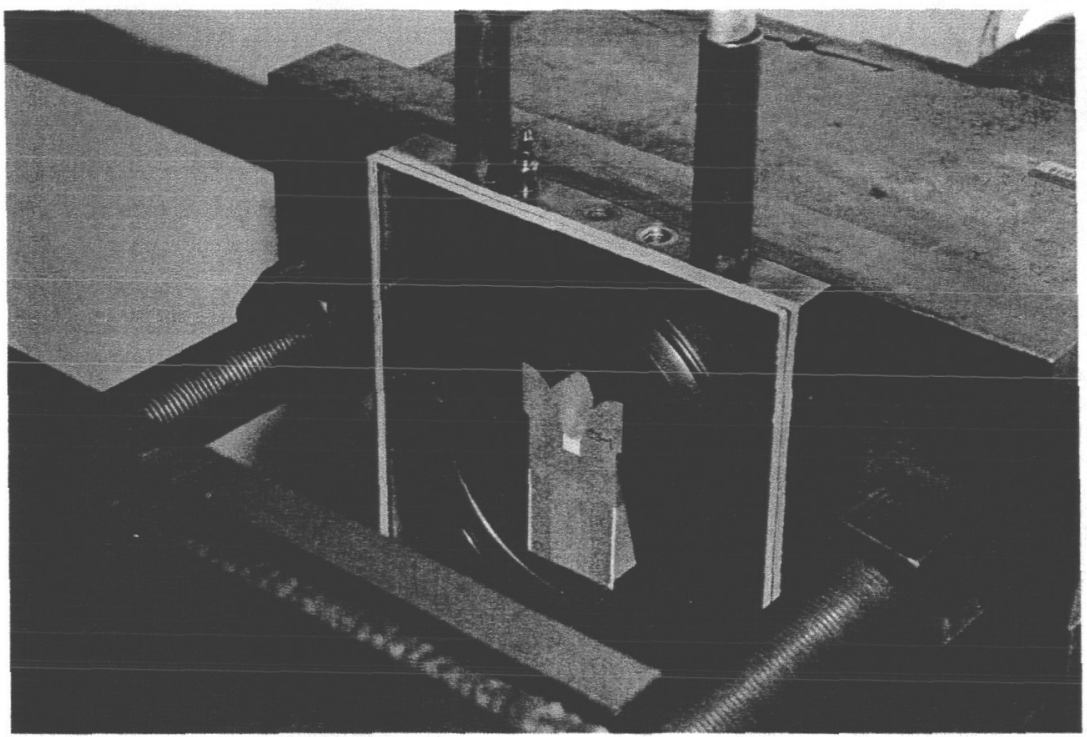

Figure 5 Crack propagation test fixture setup.

\section{Data Analysis}

\section{A. Center One-Sided Crack Specimen Data Analysis}

Data analysis of the center one-sided crack tests was accomplished by first determining the time that crack initiation occurred from the high-speed digital video. This was performed using the Phantom ${ }^{\circledR}$ high-speed digital video software. Still frames taken from the high-speed digital video of a center one-sided crack test are shown in Figure 6. Frame 1 in the series shows the specimen configured in the pressurized Instron chamber just prior to commencement of the test. Frame 2 shows the specimen being pulled in tension as crack initiation begins to occur. The left-most black vertical line, which marked the location of the initial crack tip, was used as a visual aid to assist in determining when crack initiation occurred. Frame 3 shows the specimen as the crack is propagating prior to total specimen failure.

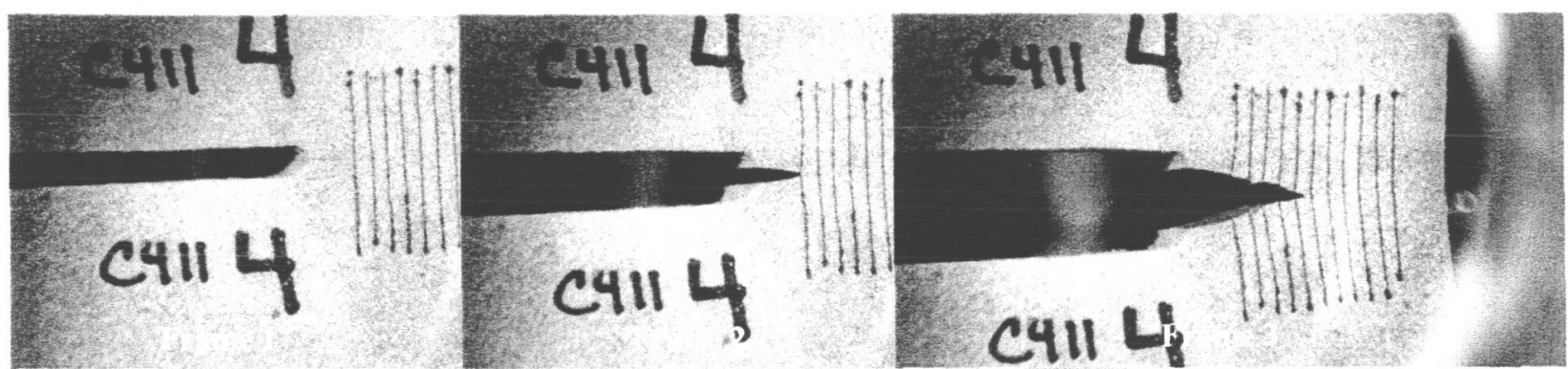

Figure 6 Video stills of center one-sided crack specimen test.

Selection of crack initiation time was a critical step in the data analysis process, as small errors in this selection could potentially result in erroneous fracture toughness values. Because of this, significant effort was placed on correct determination of the crack initiation time. Selection of the crack initiation time was performed by running the video until the crack extended just beyond the left-most black vertical line. The video was then run in reverse, and the time of crack initiation was selected. At least two engineers and/or technicians repeated this process several times for each test. 
With crack initiation time known, the load at crack initiation, or critical load, was determined. This was done through a simple correlation with the load-time trace. The high-speed digital video and the Instron were synchronized such that $\mathrm{t}=0$ was the same for both the video file and the load curve.

The load-displacement curve was then integrated according to Eq. (1) to determine the fracture toughness of the specimen.

$$
G_{c}=\frac{\eta}{B} \cdot \int_{0}^{\delta_{c}} P \cdot d \delta
$$

The geometry factor $\eta$ was determined analytically in an ABAQUS ${ }^{\circledR}$ FE model using an arbitrary displacement with a set of constitutive material properties.

\section{B. Crack Propagation Chamber Data Analysis}

Data analysis of the crack propagation chamber specimen was performed by first determining the time to crack initiation from the high-speed digital video. As with the center one-sided crack specimen data analysis, this was accomplished using the Phantom high-speed digital video software. Figure 7 shows a series of still frames taken from a cold-gas crack propagation chamber test. Frame 1 shows the crack propagation chamber specimen loaded in the window bomb test fixture just prior to pressurization of the specimen. The white markings around the crack and on the edges of the specimen were used as visual aids to assist in high-speed video analysis. The bottom edge of the white square around the crack marked the location of the initial crack tip, and helped determine when crack initiation occurred. The thin white lines on the edges of the propellant face were used to locate the propellant edges and ensure that proper specimen deformation occurred during pressurization. Frame 2 shows the specimen during pressurization as crack initiation just begins to occur. As can be seen in this frame, the propellant has deformed and is partially filling the specimen holder free volume, generating energy at the crack tip. Frame 3 shows the specimen as pressurization continues; the propellant has deformed further and the crack is extending.

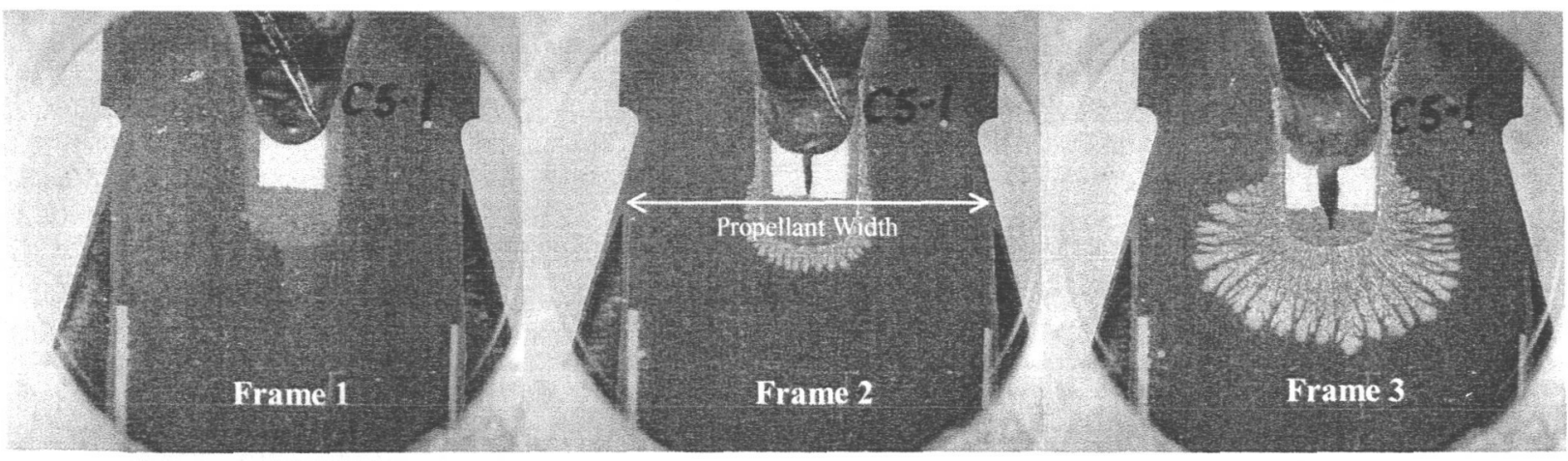

Figure 7 Video stills of cold-gas crack propagation chamber test.

The "fan" shape visible on the sides and below the crack in Frames 2 and 3 is a result of pressurizing the grease interface between the propellant and the acrylic window. As the propellant specimen deformed and filled the specimen holder free volume, the propellant near the crack "necked" and became slightly thinner, allowing pressurization to occur.

Figure 8 shows a series of still frames taken from a hot-fire crack propagation chamber test. Frame 1 shows the propellant specimen loaded into the crack propagation chamber just prior to ignition of the specimen. Frame 2 shows the specimen immediately after ignition. $\mathrm{A} \mathrm{BKNO}_{3}$ pellet is visible in this frame. Frame 3 shows the specimen as the initial crack begins to fill with hot gasses and pressurize. Frame 4 shows the specimen as the crack surfaces begin to burn and crack initiation occurs. Frames 5 and 6 show the specimen at various stages of crack propagation. The brightness and contrast in the Phantom video software were adjusted as needed to best view the hot-fire test critical events.

As with analysis of the center one-sided crack tests, selection of the crack initiation time was critical to proper analysis of the crack propagation chamber specimen tests. Since loading of the specimens was performed dynamically through specimen pressurization or ignition, the pressure in the window bomb chamber was continually increasing through crack initiation and beyond. As a result, small errors in selecting the time of crack initiation could potentially result in large errors in pressure at crack initiation, or critical pressure. The sample rates of the 
pressure transducers and high-speed digital video were sufficiently high to limit the error in selecting the crack initiation time and the corresponding critical pressure.

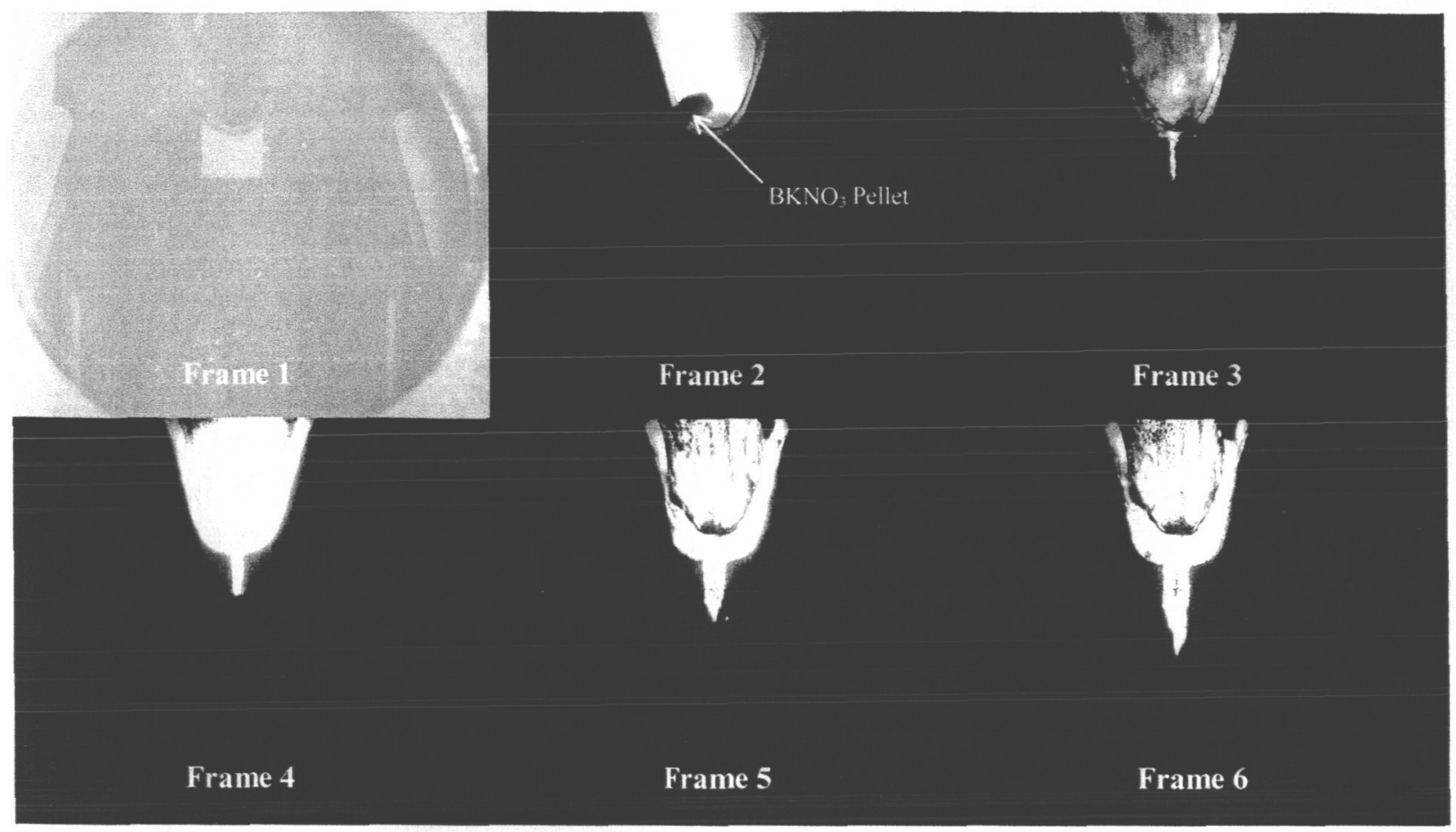

Figure 8 Video stills of the hot-fire crack propagation chamber testing.

The next step in data analysis of the crack propagation chamber specimen tests was to quantify the amount of propellant deformation that occurred at the time of crack initiation. This was done by measuring the propellant width at the crack tip at the time of crack initiation. The propellant width, shown in Frame 2 of Figure 7 , was measured using the interactive measuring tools available in the Phantom software.

The information gathered from the high-speed digital videos was used within an ABAQUS FE model to determine the fracture toughness of each specimen. The plane strain FE model for the crack propagation chamber specimen is shown in Figure 9. The same FE model was used for all crack propagation chamber testing, however, the boundary conditions along the specimen edges were adjusted as needed to simulate the different specimen holder free volumes. The "*Hyperelastic" option was used for propellant material modeling, and the nonlinear option was used for propellant geometry modeling. Because the specimen geometry was symmetric about the centerline (parallel to the

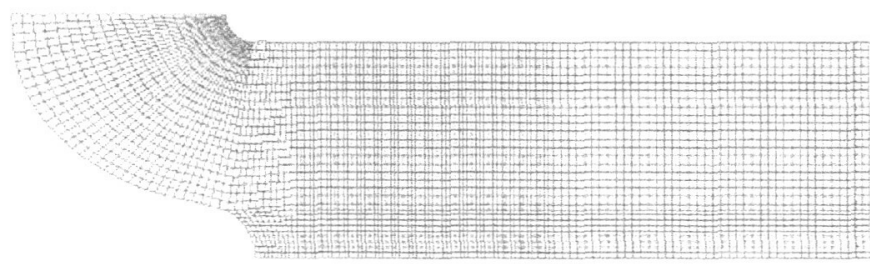

Figure 9 Crack propagation chamber specimen FE model. initial crack direction), only half of the specimen was modeled.

The actual measured pressure trace for each cold-gas and hot-fire test was input into the ABAQUS FE model, and the propellant effective modulus was varied until the specimen width in the ABAQUS FE model matched the specimen width measured from the high-speed digital video. The fracture toughness was determined by ABAQUS when the propellant deformations matched within $5 \%$. 


\section{Test Results}

\section{A. Center One-Sided Crack Specimen Test Results}

The fracture toughness of TP-H1148 propellant was successfully determined at four static pressures, two temperatures, and two crosshead displacement rates using the center one-sided crack specimen. Testing near conditions that are representative of the worst-case structural environment experienced during the RSRM ignition transient was emphasized. In general, test results followed expected trends for PBAN propellants. Fracture toughness was shown to increase with increasing rate and pressure, and decrease slightly with increasing temperature. Figure 10 graphically compares the fracture toughness of TP-H1148 propellant to the analytically predicted $\mathrm{G}_{\mathrm{i}}$ that the transition region MDFS would experience during RSRM ignition. The data have been exponentially curve fit to estimate fracture toughness between tested conditions. The exponential curve was used based on historical fracture toughness trending experience with other PBAN propellants.

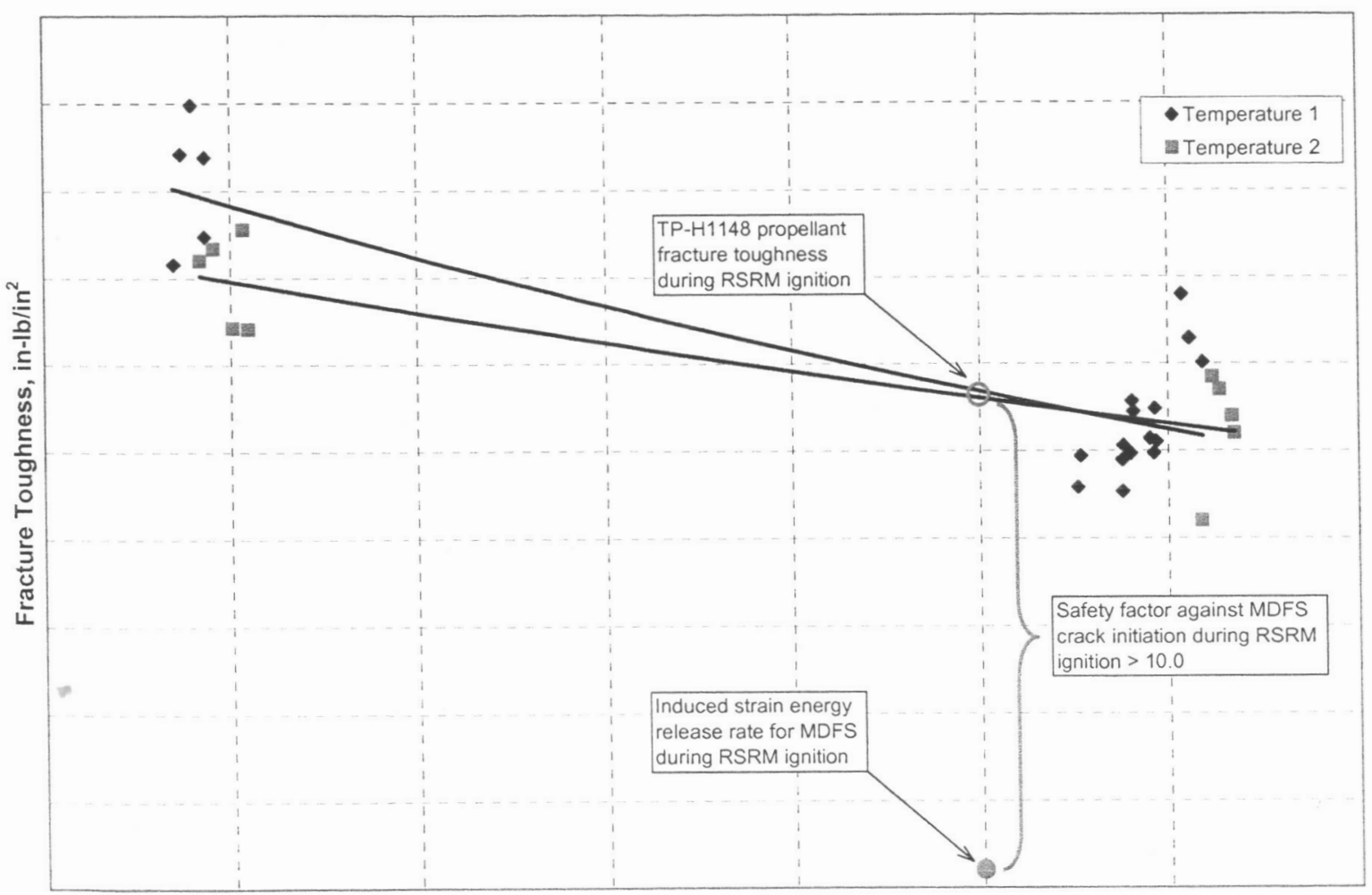

$\log (t)$, minutes

Figure 10 Fracture toughness of TP-H1148 propellant during RSRM ignition.

A safety factor against crack initiation of greater than 10 is predicted, indicating that, if present, the transition region MDFS would not undergo crack initiation during RSRM ignition. The safety factor (SF) was determined according to Eq. (2).

$$
S F=\sqrt{\frac{G_{c}}{G_{i}}}
$$




\section{B. Crack Propagation Chamber Specimen Test Results}

The fracture toughness of TP-H1148 propellant was also determined by testing the crack propagation chamber specimen in the three specimen holders identified in Figure 4. Because loading of this specimen was performed through dynamic pressurization to simulate the RSRM ignition transient, the pressure at crack initiation was not a controlled variable. Rather, the pressure at crack initiation was determined through correlation of the high-speed digital video with the recorded pressure-time trace. As a result, the fracture toughness of TP-H1148 propellant using the crack propagation chamber specimen was determined at many pressures and rates.

The fracture toughness of TP-H1148 propellant determined from the crack propagation chamber specimen was $23-44 \%$ lower than the fracture toughness determined from the center one-sided crack specimen. The crack propagation chamber fracture toughness results also followed expected trends for PBAN propellants. Figure 11 shows a comparison of the fracture toughness determined from the crack propagation chamber specimen, to the center one-sided crack specimen fracture toughness and the analytically predicted $\mathrm{G}_{\mathrm{i}}$ for the transition region MDFS during RSRM ignition. Even though the fracture toughness determined by the crack propagation chamber specimen is up to $44 \%$ lower than that for the center one-sided crack specimen, the safety factor against initiation of the transition region MDFS during RSRM ignition is still greater than 8.0. This again indicates, that even if present during RSRM ignition, the MDFS would not undergo crack initiation.

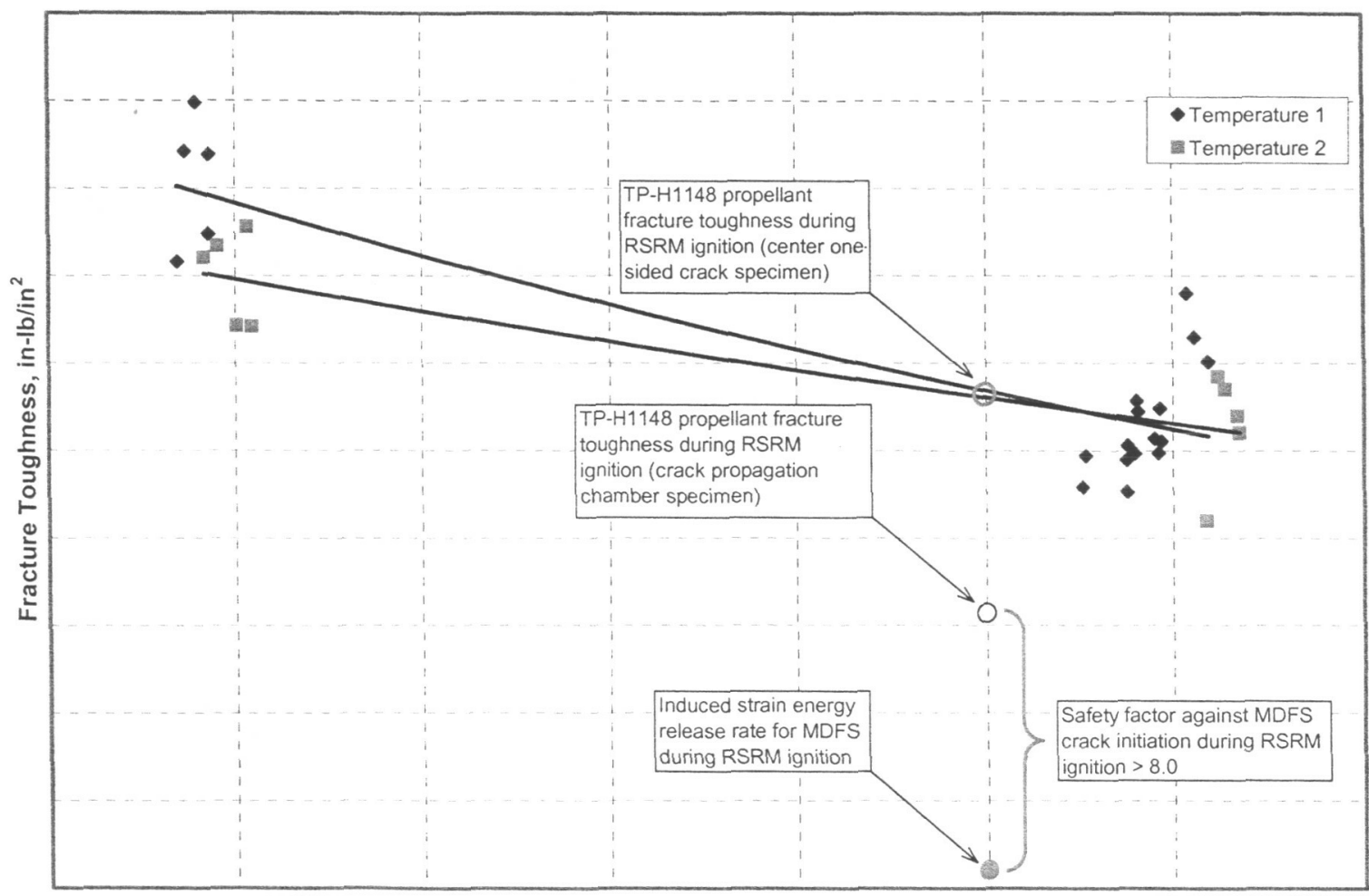

$\log (\mathrm{t})$, minutes

Figure 11 Fracture toughness of TP-H1148 propellant during RSRM ignition.

There are several possible explanations for why the fracture toughness values determined from the crack propagation chamber specimen were lower than that of the center one-sided crack specimen. The most probable explanation is the difference in loading environments under which testing was performed. In addition, the effects of friction at the acrylic window-grease-propellant interface, and pressurization loads on the face of the propellant specimen were not included in the FE model.

Crack propagation chamber testing in the fourth specimen holder demonstrated that the transition region MDFS would not initiate during RSRM ignition. None of the specimens tested in the fourth specimen holder underwent crack initiation in either cold-gas pressurization or hot-fire combustion. However, post analysis of the tests indicated that less $G_{i}$ was generated in these specimens than desired. This was partially due to the fact that the propellant specimen fully filled the specimen holder free volume faster, and at a lower pressure, than expected. 
Because of this, the full $\mathrm{G}_{\mathrm{i}}$ was not generated in the initial crack tip, and a complete simulation of the RSRM ignition environment was not accomplished.

\section{Conclusion}

Fracture toughness testing using two independent test specimens conclusively show that the RSRM forward segment transition region MDFS will not initiate during RSRM ignition. The fracture toughness of the propellant greatly exceeds the analytically predicted induced strain energy release rate of the MDFS. Although there has never been strain-induced cracks observed in the RSRM forward segment transition region propellant, nor would this test series ever be used to justify the presence of cracks, results of this testing provide significant confidence concerning the capability of TP-H1148 propellant to withstand initiation and propagation of the MDFS during RSRM ignition.

\section{Acknowledgments}

This test effort was performed under NASA contract number NAS8-97238, under the direction of Marshall Space Flight Center. The author wishes to thank the following individuals for their contributions to this test effort: Jon Janssen, Karl Kersker, Kirk Miller, Mike Dixon, Russ Stuart, Casey Taylor, Travis Hendrickson, and Mike Bunn.

\section{References}

${ }^{1}$ Critical Defect Assessment Program, ATK Thiokol Internal Report

2"Structural Ballistic Risk Assessment Methodology Final Report”, PL-TR-95-3028, Air Force Phillips Laboratory, Edwards AFB, CA, November 1995 . 